\title{
A Stable Stieltjes Technique for Computing Orthogonal Polynomials and Jacobi Matrices Associated With a Class of Singular Measures Constructive Approximation 12, (1996) 509-530.
}

\author{
Giorgio Mantica \\ Istituto di Scienze Matematiche, Università di Milano a Como, \\ Via Lucini 3, I-22100 COMO, ITALY \\ MANTICA@MI.INFN.IT \\ This paper is dedicated to the memory of Prof. Joseph Ford \\ 1991 Mathematics Subject Classification: 28A80, 58F11, 65D30 \\ Keywords and Phrases: Self-similar measure, iterated function system, \\ orthogonal polynomial, Jacobi matrix, Lanczos technique
}

\begin{abstract}
A recursive technique for the determination of Jacobi matrices associated with multifractal measures generated via Iterated Functions Systems is described. This technique allows for the stable determination of large-rank matrices, a task for which the conventional approach, classical polynomial sampling, is proven here to be severely ill-conditioned.

Application to the integration of smooth functions is presented in a physical example, and relevance of this new technique to the study of the asymptotic properties of orthogonal polynomials of singular measures is discussed. Further applications to the study of almost periodic quantum systems are briefly mentioned.
\end{abstract}

\section{Introduction}

Among the nicest examples of measures on the real line we can count those for which moments exist, and uniquely determine the measure. They give rise to what is called a determined moment problem [1,2]. A wide variety of techniques has been developed to solve this problem, that is to say, to recover the original measure from the knowledge of its moments. Solving for a measure may have different meanings. For us, it will be equivalent to determine the (tridiagonal) Jacobi matrix which translates the recurrence relations of the associated system of orthonormal polynomials. In fact, from this information one can immediately derive Gaussian summation formulae for integrals of sufficiently smooth functions, and integration is what measures are for. 
We shall outline in the paper other well-known reasons behind this prominent rôle of orthogonal polynomials.

Moments are not the sole source of information that one might want to consider in this quest for the Jacobi matrix. In fact, significant progress in this field has been obtained by admitting usage of the larger class of operations called polynomial sampling: The common hypothesis is made that one is able to compute-whether experimentally or theoretically-the integrals of suitable polynomials, whose degree ranges from zero to a certain maximum. For instance, Chebyshev polynomials have played with success the rôle of sampling polynomials in many instances, and efficient algorithms have been designed for this scope $[3,4,5,6]$.

Nevertheless, sampling with classical orthogonal polynomials cannot be a procedure of general purpose, and there exists a wide class of measures for which it turns out to be severely ill-conditioned. We shall prove that this class consists of measures supported on a finite interval (hence, leading trivially to a determined moment problem) but possessing a singular, fractal character. The proof unveils the mechanism behind the exponential divergence of the conditioning number of this problem, and poses a serious challenge to our capability of dealing with these objects.

A first example in this class is offered by the equilibrium measure of the Julia set of $P(z)=z^{2}-\lambda$, which, for $\lambda \geq 2$, is supported on the real axis. However, the algebraic properties of these measures permit to compute the Jacobi matrix via a (stable) recurrence algorithm parameterized by $\lambda[7,8]$, (valid also for polynomials of higher degree [9]).

In this paper we consider the wider class of equilibrium measures of linear Iterated Functions Systems, I.F.S., characterized by affine scaling relations, whose parameters can be chosen appropriately, in order to approximate arbitrarily well any measure [10]. Since they also permit the efficient encoding of these objects, the related approximation problem $[11,12,13]$ is endowed with important practical applications. The determination of the associated Jacobi matrices cannot be performed here by algebraic means, and therefore constitutes a challenging and interesting problem, which is solved in this work by means of a Stieltjes-like technique.

The plan of the paper is the following: In the next section we introduce the formalism of I.F.S. A few basic notions on orthogonal polynomials follow in Sect. III. Sections II and III are review, and can be skipped by the reader already acquainted with theory and notations. In Sect. IV we discuss the ill-conditioning of classical polynomial sampling when applied to singular measures, illustrating the theory with numerical examples. Sect. V is the heart of the paper: We explain a constructive procedure for the computation of Jacobi matrices associated with I.F.S. balanced measures. The stability of this procedure is proven in Sect. VI, and its numerical performance, compared to that of classical polynomial sampling is tested in a number of significant cases in Sect. VII. A first application of our results is presented in Sect. VIII, where we discuss the integration of continuous functions with respect to fractal measures. The Conclusions summarize the work and we anticipate further investigations. 


\section{$2 \quad$ Linear Iterated Functions Systems}

The class of singular measures studied in this paper is generated by systems of iterated functions (I.F.S.) $[14,15,16,17]$. An I.F.S. is a finite collection of mappings

$$
\phi_{i}: K \rightarrow K, i=1, \ldots, M,
$$

on a compact metric space $K$, with associated probabilities $\pi_{i} ; \pi_{i}>0, \sum_{i} \pi_{i}=1$. In this paper $K$ will be the $[0,1]$ interval of the real line. We denote by $d$ the distance in $K$. The maps $\left\{\phi_{i}\right\}$ are required to be contractive: there exist positive constants $\epsilon_{i}$ such that

$$
d\left(\phi_{i}(x), \phi_{i}(y)\right) \leq \epsilon_{i} d(x, y), \quad \forall x, y \in K, \forall i=1, \ldots, M,
$$

with $\epsilon_{i}<1$, to ensure the uniqueness properties that we shall need in the following.

These maps allow us to construct a measure on $K$ with given similarity properties, extending a procedure already present in Zygmund [18]. This can be done in two steps. First, we consider the unique solution of the equation

$$
A=\bigcup_{i=1, \ldots, M} \phi_{i}(A),
$$

in $\mathcal{P}(K)$, the set of all subsets of $K$. The set $A$ is invariant under the action of shrinking it to smaller copies of itself, and glueing these copies together. Second, we define a stochastic process for which $A$ is the attractor: This is the discrete-time Markov process on $K$ with transition probabilities $P(x, B)$ :

$$
P(x, B)=\sum_{i=1}^{M} \pi_{i} \chi_{B}\left(\phi_{i}(x)\right),
$$

where $B$ is a Borel subset of $K$ and $\chi_{B}(x)$ is its characteristic function. A realization of this process can be obtained starting from an initial point $x \in K$, and applying repeatedly a mapping $\phi_{\sigma}$ chosen each time in a random, independent fashion from the full set of maps, with probability $\pi_{\sigma}$. The images of $x$ rapidly converge to, and wander on, the attractor $A$ defined by eq. (3). The equilibrium measure induced by (4) on $A$ is the singular, multi-fractal measure we are about to study. Because of eq. (4), this measure is equivalently defined by the "balance" property

$$
\int_{K} f d \mu=\sum_{i=1}^{M} \int_{K} \pi_{i}\left(f \circ \phi_{i}\right) d \mu,
$$

holding for any continuous function $f$ on $K$. This equation is going to be a crucial tool in our investigation.

A convenient choice of the maps $\phi_{i}$ is that of linear affine transformations, for which we adopt the notation

$$
\phi_{i}(x)=\delta_{i} x+\beta_{i}, \quad i=1, \ldots, M,
$$

where $\delta_{i}, \beta_{i}$ are real constants and the contraction rates $\delta_{i}$ have modulus less than one. Without loss of generality, we shall always assume that $\phi_{1}(0)=0$, and that the support of $\mu$ is included 
in $[0,1]$. Linear I.F.S. permit a large number of formal manipulations. One of these is the recursive determination of the moments of the balanced measure $\mu$, which can be derived choosing $f(x)=x^{n}$ in eq. (5):

$$
\mu_{n}=\int_{K} x^{n} d \mu(x)=\int_{K} \sum_{i=1}^{M} \pi_{i}\left(\sum_{j=0}^{n}\left(\begin{array}{l}
n \\
j
\end{array}\right) x^{j} \delta_{i}^{j} \beta_{i}^{n-j}\right) d \mu=\sum_{i=1}^{M} \pi_{i} \sum_{j=0}^{n}\left(\begin{array}{l}
n \\
j
\end{array}\right) \delta_{i}^{j} \beta_{i}^{n-j} \mu_{j} .
$$

Eqs. (7) are a triangular set of relations which permit us to determine $\mu_{n}$ recursively. (A theory for the determination of the moments of non-linear I.F.S. has been developed by Vrscay [19].)

In the case of disconnected I.F.S. (i.e. those for which the sets at r.h.s. of eq. (3) do not intersect each other) the multi-fractal properties of I.F.S. balanced measures can be easily computed: The spectrum of generalized dimensions $D_{q}$ follows from the equation

$$
\sum_{j=1}^{M} p_{j}^{q} \delta_{j}^{-\tau}=1
$$

whose unique solution defines $\tau$ as a function of $q$. One then obtains $D_{q}=\frac{\tau(q)}{1-q}$. From eq. (8) one is able to tailor the map parameters in order to obtain various multi-fractal spectra. Therefore, the relative simplicity of linear I.F.S. balanced measures makes them an optimal tool for the study of singular measures on the real line. We shall now set the stage to investigate the set of associated orthogonal polynomials.

\section{Orthogonal Polynomials and Jacobi Matrices}

According to Gautschi [3], the determination of the Jacobi matrix associated with a given measure is a fundamental problem. In fact, knowledge of the Jacobi matrix enables one to: i) build recursively the complete system of associated orthogonal polynomials, ii) evaluate orthogonal sums via the Clenshaw's algorithm [20], and iii) obtain weights and locations for Gaussian points, with immediate application to numerical quadratures. Let us briefly recall these facts, mainly to fix notations.

The set of orthonormal polynomials $\left\{p_{n}\right\}_{n \in \mathcal{N}}$ w.r.t. a positive measure $\mu$ is defined by the relations:

$$
\int p_{j}(x) p_{l}(x) d \mu(x)=\delta_{j, l}
$$

where $\delta_{j, l}$ is the Kronecker delta. Orthonormal polynomials satisfy a three-term recurrence relation

$$
x p_{j}(x)=r_{j+1} p_{j+1}+A_{j} p_{j}(x)+r_{j} p_{j-1}(x),
$$

initialized by

$$
p_{-1}=0, \quad p_{0}=1
$$

from which the sequence $p_{n}(x)$ can be computed, when the real coefficients $A_{j}, r_{j} \geq 0$ are known. 
The Jacobi matrix $J$ is constructed as the real, symmetric, tridiagonal matrix whose diagonal and outer diagonals are the vectors $A_{j}$ and $r_{j}$, respectively. The non-zero entries of $J$ are therefore

$$
J_{i, i}=A_{i}, \quad J_{i+1, i}=J_{i, i+1}=r_{i+1}, \quad i=0,1, \ldots
$$

In terms of the Jacobi matrix, the recurrence relation (10) can be written as $J p(x)=x p(x)$, where $p(x)$ is the vector whose components are the orthonormal polynomials evaluated at site $x$.

While $J$ is an operator in $l_{2}$, the space of square summable sequences, we shall indicate by $J_{N}$ the $N$-dimensional truncation of $J$. This finite-rank matrix is in direct relation with the zeros of the orthogonal polynomial $p_{N}(x)$. In fact, if $\bar{x}$ is one of these, the $N$ dimensional vector $\left(p_{0}(\bar{x}), p_{1}(\bar{x}), \ldots, p_{N-1}(\bar{x})\right)$ is an eigenvector of $J_{N}$ of eigenvalue $\bar{x}$. Hence, the $N$ distinct zeros of $p_{N}(x)$, denoted by $x_{l, N}, l=1, \ldots, N$, compose the full spectrum of $J_{N}$, and can be computed accordingly.

Diagonalization of $J_{N}$ permits the calculation of the so-called Christoffel numbers, $w_{l, N}$, required in Gaussian quadrature formulae: they become the squares of the first component of the normalized eigenvectors. Gauss first formula, which we shall use in the following, reads:

$$
\int_{0}^{1} f(x) d \mu=\sum_{l=1}^{N} w_{l, N} f\left(x_{l, N}\right)+R .
$$

If $f$ has the required regularity properties, the remainder $R$ can be simply expressed as $R=$ $\frac{f^{(2 N)}(\xi)}{k_{N}^{2} 2 N !}, k_{N}=1 /\left(r_{1} r_{2} \ldots r_{N}\right)$, and $\xi \in(0,1)$. A second Gauss formula can also be derived, which combined with the first leads sometimes to the determination of upper and lower bounds to the integral (13)-notably when $f$ is a totally monotonic function. Numerical integration can therefore be reduced to the diagonalization of the Jacobi matrix $J_{N}$. We shall return to this point in Section VIII, when we discuss the integration of smooth functions w.r.t. a fractal measure.

\section{Classical Polynomial Sampling of Singular Measures}

The linear character of the I.F.S. maps $\phi_{i}$ defined above permits the recursive evaluation of the moments $\mu_{n}$, eq. (7), and, more generally, of the modified moments $\sigma_{n}=\int q_{n}(x) d \mu$, where $\left\{q_{n}(x)\right\}$ is any set of polynomials, not necessarily orthogonal, but linked by a threeterm recurrence relation: $x q_{n}=\alpha_{n} q_{n+1}+\beta_{n} q_{n}+\gamma_{n} q_{n-1}$. Modified moments are the basis of a computational procedure to evaluate the Jacobi matrix which mainly consists of a Cholewsky decomposition of the Gram matrix associated with the set $\left\{q_{n}\right\}[4,5,6]$. For measures supported on an interval, a common choice for the set $\left\{q_{n}\right\}$ is that of Chebyshev polynomials of the first kind. Other classical polynomials can also be employed, whence the name of classical polynomial sampling, to be distinguished from the standard technique (sampling with ordinary moments). Classical polynomial sampling leads to well-conditioned algorithms for a wide class of measures, and it is usually superior to the standard technique [3], but (despite some progress reported in [21]) it is almost as bad as the latter when applied to singular measures, as we shall now prove. 
The construction of Gaussian quadratures from modified moments has been studied in depth by Gautschi [3]: He performed the stability analysis of the set of equations

$$
\sigma_{k}:=\int q_{k}(x) d \mu(x)=\sum_{i=1}^{n} w_{i, n} q_{k}\left(x_{i, n}\right), \quad k=0,1, \ldots, 2 n-1
$$

where $\left\{q_{k}\right\}$ are sampling orthogonal polynomials, associated with a sampler measure $\sigma$, in principle different from the sampled measure $\mu$. These equations follow from the Gaussian summation formulae (13), which are exact for polynomials of degree strictly less than $2 n$. In eq. (14), the sampler measure is known, as well as its orthogonal polynomials and the moments $\sigma_{k}$. The unknowns are the Gaussian points and the Christoffel numbers (also called Gaussian weights). We take them to be the entries of the $2 n$-dimensional solution vector $\left(w_{1, n}, \ldots, w_{n, n}, x_{1, n}, \ldots, x_{n, n}\right)$, which we denote by $y$. Then, the system of equations (14) can be formally written as $F(y)=\sigma$. Its relative asymptotic condition number $k_{n}$ is defined in terms of the inverse Jacobian (partial derivatives) matrix of $F, F_{y}^{-1}$ :

$$
k_{n}:=\frac{\|\sigma\|}{\|y\|}\left\|F_{y}^{-1}(y)\right\|
$$

where we can choose any convenient norm, like e.g. Euclidean: $\|y\|_{2}^{2}=\sum_{j=1}^{2 n}\left|y_{j}\right|^{2}$. The condition number so defined has a transparent meaning: Suppose that a relative error $\varepsilon$ affects the determination of the vector $\sigma$; the relative error occurring in the solution vector $y$ is then $k_{n} \times \varepsilon$. Therefore, $k_{n}$ measures the stability of any procedure leading from moments to Gaussian points and weights. Since the Jacobi matrix can be obtained from these latter via a stable procedure (and viceversa), $k_{n}$ is also an indicator of the conditioning of the determination of the Jacobi matrix starting from moments. We shall now use Gautschi's analysis to expose the failure of classical polynomial sampling when applied to singular measures.

The Jacobian matrix $F_{y}$ is readily written; it can be factored as (we shall define our terms in a moment)

$$
F_{y}=\Xi \Lambda
$$

and therefore $\left\|\Lambda^{-1} \Xi^{-1}\right\|$ determines the conditioning of the problem. We shall study separately the potentiality for ill-conditioning of these matrices. To illustrate our theory, we shall consider three different measures on $[-1,1]$ :

- The orthogonality measure of Chebyshev polynomials of the first kind, $d \sigma=\frac{1}{\pi \sqrt{1-x^{2}}} d x$;

- The orthogonality measure of Legendre polynomials, $d \sigma=\frac{1}{2} d x$;

- The balanced measure of the I.F.S. with $M=2, \delta_{1}=\delta_{2}=\frac{2}{5}, \beta_{1}=0, \beta_{2}=\frac{3}{5}, \pi_{1}=\pi_{2}=\frac{1}{2}$, which can be dilated and translated so to be symmetrical on $[-1,1]$.

The numerical computations involving the third measure are only possible employing the stable solution algorithm to be presented in the next section.

Let us first examine the matrix $\Lambda$, which is simply defined in terms of the Christoffel numbers, $w_{i, n}$, by

$$
\Lambda=\operatorname{diag}\left(1, \ldots, 1, w_{1, n}, w_{2, n}, \ldots, w_{n, n}\right) .
$$


Since $\sum_{i} w_{i, n}=1$, the norm of $\Lambda^{-1}$ is piloted by the non-uniformity of the distribution of $w_{i, n}$, and a large value of this norm reveals the presence of Gaussian points of low weight. Quite evidently, this is a characteristic of the sampled measure alone. In Fig. 1 we show the Euclidean norms of the inverse $\Lambda$ matrices associated with the singular and the two classical measures introduced above. The power-law behaviour of the classical examples contrasts sharply with the exponential growth of the singular case. We have an explicit rule for the Chebyshev norm: $\left\|\Lambda^{-1}\right\|_{2}=\left(n+n^{3}\right)^{1 / 2}, n$ being the matrix dimension, and we can explain-at least qualitativelythe reason for the other behaviour.

In fact, from a Green function argument we know that $p_{n}(x)$ grows exponentially in $n$ when $x$ lies in one of the gaps in the support of the singular measure. Now, Gaussian points (i.e. zeros of the associated orthogonal polynomials) can as well lie in such gaps, like in the example presented in Fig. 1: odd polynomials of a symmetric measure must have a zero at the origin-here at the center of the largest gap. Let $w(0)$ be the weight associated with this point. At the same time, it must be $\int p_{l}(x) d \mu=0$ for any $l$, and for $l<2 n$ this integral can be exactly replaced by a Gaussian sum, which includes the term $p_{l}(0) w(0)$. When $l$ is even, the first factor is exponentially large in $l$, thus demanding an exponentially smaller Christoffel weight $w(0)$.

A more subtle interplay between sampled and sampling measure is at the root of the conditioning properties of the matrix $\Xi$, which is defined as follows:

$$
\Xi=\left[\begin{array}{llllll}
q_{0}\left(x_{1, n}\right) & \ldots & q_{0}\left(x_{n, n}\right) & q_{0}^{\prime}\left(x_{1, n}\right) & \ldots & q_{0}^{\prime}\left(x_{n, n}\right) \\
q_{1}\left(x_{1, n}\right) & \ldots & q_{1}\left(x_{n, n}\right) & q_{1}^{\prime}\left(x_{1, n}\right) & \ldots & q_{1}^{\prime}\left(x_{n, n}\right) \\
\ldots & \ldots & \ldots & \ldots & \ldots & \ldots \\
q_{2 n-1}\left(x_{1, n}\right) & \ldots & q_{2 n-1}\left(x_{n, n}\right) & q_{2 n-1}^{\prime}\left(x_{1, n}\right) & \ldots & q_{2 n-1}^{\prime}\left(x_{n, n}\right)
\end{array}\right]
$$

The inverse $\Xi^{-1}$ can be obtained explicitly, in terms of the fundamental Hermite interpolation polynomials $h_{l}$ and $k_{l}$ of degree $2 l-1$, belonging to the abscissae $x_{i, l}$, for $j=1, \ldots, n$, as shown in [3]. For our purposes, it is not important to reproduce here all the explicit formulae, but to report just the last, which gives the Frobenius norm of $\Xi^{-1}$ in terms of an integral with respect to the sampler measure $\sigma$ of a suitable interpolation polynomial $g_{n}(x)$ :

$$
\nu_{n}:=\left\|\Xi^{-1}\right\|_{F}^{2}=\int g_{n}(x) d \sigma(x) .
$$

The polynomial $g_{n}(x)$ is uniquely determined by the sampled measure, via

$$
g_{n}(x):=\sum_{j=1}^{n}\left[h_{j}^{2}(x)+\frac{1}{w_{j, n}^{2}} k_{j}^{2}(x)\right] .
$$

Quoting Gautschi, the magnitude of $g_{n}(x)$ on the support of $\sigma$ determines the Frobenius norm of $\Xi^{-1}$. Indeed, we think that we get a better picture of what is going on if we turn this statement around, and we say that what enhances-sometimes dramatically-the norm of $\Xi^{-1}$ is the magnitude of the measure $\sigma$ outside the support of $\mu$. In fact, $g_{n}(x)$ is positive, and it is constrained on the Gaussian points of $\mu$ by:

$$
g_{n}\left(x_{i, n}\right)=1, \quad g_{n}^{\prime}\left(x_{i, n}\right)=0 .
$$


Therefore, $g_{n}$ is order of unity on the spectrum of $\mu$, the sampled measure. To the contrary, it tends to blow up (like all interpolating polynomials) in the regions not populated by Gaussian points, reaching values which are larger the wider the intermediate regions between such points and the higher the polynomial order $n$. Now, we must obviously think of $g_{n}$ as fixed, since it depends on the measure we are about to sample, while we are at freedom of choosing $\sigma$, the sampling measure. This has the following effect.

On the one hand, measures associated with classical polynomials (e.g. Chebyshev) are absolutely continuous, their Gaussian points are smoothly distributed on the interval supporting the measure, and they give rise to polynomials $g_{n}$ with nicely uniform oscillations, like those plotted in Fig. 2. On the other hand, Cantor-like supports of singular measures are characterized by gaps at all scales, Gaussian points crowd sets of zero Lebesgue measure, and the related $g_{n}$ polynomials reach large amplitudes in the gaps, as one can see from Fig. 3. Increasing the degree $n$ enhances two strictly related phenomena: oscillations in the large gaps increase dramatically, and smaller gaps are "discovered" and pop out. Therefore, the interplay between the smooth distribution of Gaussian points of the sampler measure, and the oscillations of $g_{n}$ polynomials of the singular sampled measure makes us expect an exponential divergence of the integrals (19) with respect to $n$. Notice that, again, in the case of the opposite combination (singular sampling of classical measures), no ill-conditioning is predicted!

This theory can be verified by direct numerical computation: in Fig. 4 we plot $\nu_{n}$, the Frobenius norms of $\Xi^{-1}$, as a function of $n$, when a classical measure is sampled by another classical measure or by the singular I.F.S. measure. In all cases the norm is bounded and the problem is well-conditioned. In Fig. 5 the singular measure is sampled by the classical measures. We notice an exponential increase of $\nu_{n}$, which starts at values of $n$ of about ten.

To end this section, we can summarize our results as follows: We expect that classical polynomial sampling of any measure supported on a Cantor set is ill-conditioned. This is manifest in the exponential divergence of the norms of both $\Lambda^{-1}$ and $\Xi^{-1}$. On the contrary, sampling of classical measures via "singular" polynomials does not show any symptom of potential illconditioning. Having diagnosed the illness, let us now turn to a cure.

\section{Recursive Computation of the Jacobi Matrix for Linear I.F.S.}

In the preceding section we have seen that ill-conditioning of classical polynomial sampling is caused by the fact that the measure associated with the sampling polynomials (say, Chebyshev) and the sampled measure are mutually singular. On the contrary, the former ought to be supported on the same set of the latter, to provide an effective sampling. Hence, one might think of using the very same orthogonal polynomials of the singular measure to do the sampling. Since these polynomials are the unknowns of the problem, the idea is at least imaginative. Nevertheless, the simple rescaling properties of I.F.S. measures permit us to get around this problem, via an algorithm which after the fact resembles that originally proposed by Stieltjes 
in 1884 [22], quoted in [3]: The recurrence relations (10) imply that

$$
A_{n}=\int p_{n}(x) x p_{n}(x) d \mu ; r_{n}=\int p_{n-1}(x) x p_{n}(x) d \mu .
$$

We shall try to determine directly the integrals in (22): This is the essence of the Stieltjes approach.

To achieve this goal, a simple but crucial observation is contained in the following Lemma:

Lemma 1 For any $n$,

$$
p_{n}\left(\phi_{i}(x)\right)=\sum_{l=0}^{n} \Gamma_{i, l}^{n} p_{l}(x)
$$

and the coefficients $\Gamma_{i, l}^{n}, l=0, \ldots, n$ can be determined recursively from the map parameters $\delta_{i}, \beta_{i}, \pi_{i}$, for $i=1, \ldots, M$ and from the Jacobi matrix entries $A_{j}$, for $j=0,1, \ldots, n-1$, and $r_{m}$, for $m=0,1, \ldots, n$.

Proof. It is immediate that the $n$-th degree polynomials $p_{n}\left(\phi_{i}(x)\right)$ can be expanded on the first $n$ orthogonal polynomials, with coefficients denoted by $\Gamma_{i, l}^{n}$. The recursive determination of the $\Gamma$ 's can be simply proven by induction, showing in the passage that the highest order polynomial, $p_{n}$, appears always in these manipulations in the form of the product $r_{n} p_{n}$.

Because of the last observation, the coefficients in the expansion of $r_{n} p_{n}$ can be determined without knowing $r_{n}$. Let $\tilde{p}_{n}(x)=r_{n} p_{n}(x)$ be this polynomial. We can therefore write a second decomposition:

$$
\tilde{p}_{n}\left(\phi_{i}(x)\right)=\tilde{\Gamma}_{i, n}^{n} \tilde{p}_{n}(x)+\sum_{l=0}^{n-1} \tilde{\Gamma}_{i, l}^{n} p_{l}(x),
$$

where the coefficients $\tilde{\Gamma}$ can be computed recursively as in Lemma 1 , on the basis of the knowledge of only $A_{j}, r_{j}$, for $j=0,1, \ldots, n-1$. We shall make use of this fact in the following.

Let us now focus our attention on the non-diagonal entries of the Jacobi matrix:

Lemma 2 For any $n$, the coefficient $r_{n}$ can be determined from the coefficients in the expansions (23) of order $n-1$, and (24) of order $n$, from the map parameters $\delta_{i}, \beta_{i}, \pi_{i}$, for $i=1, \ldots, M$, and from the Jacobi matrix entries $A_{j}, r_{j}$, for $j=0,1, \ldots, n-1$.

Proof. From eq. (22) we can write

$$
r_{n}^{2}=\int \tilde{p}_{n}(x) x p_{n-1}(x) d \mu .
$$

Hence, using the balance eq. (5) and Lemma (1) this becomes (when not specified, integrals are taken w.r.t. $d \mu$ )

$$
r_{n}^{2}=\sum_{i=1}^{M} \pi_{i} \int\left(\delta_{i} x+\beta_{i}\right)\left[\sum_{m=0}^{n-1} \sum_{l=0}^{n-1} \tilde{\Gamma}_{i, m}^{n} \Gamma_{i, l}^{n-1} p_{m}(x) p_{l}(x)+\sum_{l=0}^{n-1} \tilde{\Gamma}_{i, n}^{n} \Gamma_{i, l}^{n-1} \tilde{p}_{n}(x) p_{l}(x)\right] .
$$


We use the recurrence relations (10), to get

$$
r_{n}^{2}=\sum_{i=1}^{M} \pi_{i}\left(B_{i}+C_{i}+D_{i}\right)
$$

where we have put:

$$
\begin{gathered}
B_{i}=\sum_{l=0}^{n-1}\left(\beta_{i}+\delta_{i} A_{l}\right) \tilde{\Gamma}_{i, l}^{n} \Gamma_{i, l}^{n-1}, \\
C_{i}=\delta_{i} \sum_{l=0}^{n-2} r_{l+1}\left(\tilde{\Gamma}_{i, l}^{n} \Gamma_{i, l+1}^{n-1}+\tilde{\Gamma}_{i, l+1}^{n} \Gamma_{i, l}^{n-1}\right),
\end{gathered}
$$

and

$$
D_{i}=\delta_{i} \tilde{\Gamma}_{i, n}^{n} \Gamma_{i, n-1}^{n-1} r_{n}^{2} .
$$

Because of contractivity of the maps, $\left|D_{i}\right| r_{n}^{-2}<1$, and $r_{n}^{2}$ (and hence $r_{n}>0$ ) can be determined from eqs. (25-30) as promised.

A similar Lemma holds for the diagonal entries $A_{n}$ :

Lemma 3 For any $n$, the coefficient $A_{n}$ can be determined from the coefficients in the expansion (23) of order $n$ fixed, from the map parameters $\delta_{i}, \beta_{i}, \pi_{i}$, for $i=1, \ldots, M$, and from the Jacobi matrix entries $A_{j}$, for $j=0,1, \ldots, n-1$, and $r_{m}$, for $m=0,1, \ldots, n$.

Proof. We use eq. (22) and the balance relation (5):

$$
A_{n}=\int x p_{n}^{2}(x)=\sum_{i=1}^{M} \pi_{i} \int\left(\delta_{i} x+\beta_{i}\right) p_{n}^{2}\left(\delta_{i} x+\beta_{i}\right)=\sum_{i=1}^{M} \pi_{i} \int\left(\delta_{i} x+\beta_{i}\right) \sum_{m, l=0}^{n} \Gamma_{i, l}^{n} \Gamma_{i, m}^{n} p_{l}(x) p_{m}(x) .
$$

Using the orthonormality properties of the sequence $p_{l}$, and the recurrence relation, we get

$$
A_{n}=\sum_{i=1}^{M} \pi_{i}\left[\sum_{m=0}^{n}\left(\Gamma_{i, m}^{n}\right)^{2}\left(\beta_{i}+\delta_{i} A_{m}\right)+\sum_{m=0}^{n-1} \Gamma_{i, m}^{n} \Gamma_{i, m+1}^{n} \delta_{i}\left(r_{m}+r_{m+1}\right)\right],
$$

whence $A_{n}$ can be determined, being $\sum_{i=1}^{M} \pi_{i} \delta_{i}\left(\Gamma_{i, n}^{n}\right)^{2}<1$.

These Lemmas enable us to prove our main result:

Theorem 1 The Jacobi matrix associated with a balanced I.F.S. measure can be computed recursively, from the sole knowledge of the map parameters $\delta_{i}, \beta_{i}$, and $\pi_{i}$, for $i=1,2, \ldots, M$, where $M$ is the number of maps.

Proof. The recursive algorithm is structured as follows:

A Initialization. At the first step, we have $A_{0}=\mu_{1}, r_{0}=0, \Gamma_{i, 0}^{0}=1$. 
B Iteration. Suppose that we know $A_{l}, r_{l}$, and $\Gamma^{l}$ for $l=0,1, \ldots, n-1$. Then, we act as follows:

i Computation of $\tilde{\Gamma}^{n}$. We use the observation following Lemma 1.

ii Computation of $r_{n}$. We use Lemma 2.

iii Computation of $\Gamma^{n}$. Once $r_{n}$ is known, $\Gamma^{n}$ is determined easily from $\tilde{\Gamma}^{n}$.

iv Computation of $A_{n}$. We use Lemma 3. Then, we loop back to i.

It is convenient to visualize the quantities which are produced step by step in this iterative procedure:

$$
\left(\begin{array}{cc}
\Gamma^{n-1}, & r_{0}, \ldots, r_{n-1} \\
A_{0}, \ldots, A_{n-1}
\end{array}\right) \Rightarrow \tilde{\Gamma}^{n} \Rightarrow r_{n} \Rightarrow \Gamma^{n} \Rightarrow A_{n} \Rightarrow\left(\begin{array}{cc}
\Gamma^{n}, & r_{0}, \ldots, r_{n} \\
& A_{0}, \ldots, A_{n}
\end{array}\right)
$$

In the forecoming sections we shall analyze the numerical stability of the recursive algorithm versus the classical procedures. Before doing that, though, we can appreciate the beauty of the set of orthogonal polynomials which can be generated by this algorithm. In Fig. 6 we draw the eigth orthogonal polynomial of the singular measure introduced in the preceding section. Horizontally, a a finite-resolution rendering of its fractal support is plotted. Also illustrated is the beginning of the sequence $r_{n}$, with its typical almost-periodic oscillations.

\section{Stability Analysis of the Stieltjes Algorithm}

We shall now prove the numerical stability of the algorithm just presented. The proof is based on the equivalence of our technique with the well-known Lanczos' method for finding a tridiagonal form of a given operator [23], and on a technical paper by Paige [24] which discusses the numerical stability of different implementations of the latter. The proof will be divided in two steps. In the first, we show that the general structure of our technique coincides with the (stable) $A(1,7)$ version discussed by Paige. In the second, we render the correspondence explicit, reporting the matrix algebra which underlies our computations and establishing its stability, on the basis of Paige's results.

Equivalence of Lanczos' algorithm and the recursive construction of orthogonal polynomials can be seen as follows. Let $X$ be the multiplication operator by $x$ in the space $L_{2}([0,1], d \mu(x))$ of square summable functions on $[0,1]$ w.r.t. the measure $\mu$. Lanczos' technique is basically an orthogonalization of the repeated applications of $X$ on the so-called Lanczos' vectors $v_{j}$, the first of which can be chosen as wished. We let $v_{0}$ be the polynomial $p_{0}$. Then let us follow the sequence of steps of Paige's $A(1,7)$. Eq. (P1) in Paige's paper is

$$
A_{j}=\left(v_{j}, X v_{j}\right), \quad(P 1)
$$

where brackets indicate the scalar product, and we have slightly adapted the notation. Clearly, if $v_{j}$ is the $j$-th orthogonal polynomial, eq. (P1) is eq. (31), computed in Lemma 3 . This is indeed the case, because of Paige's equations (P3) and (P8), which can be combined (see Paige's eq. (P9)) to give

$$
r_{j+1} v_{j+1}=X v_{j}-A_{j} v_{j}-r_{j} v_{j-1} .
$$


The product $r_{j+1} v_{j+1}$ is the vector $w_{j+1}$ in Paige's notation, and the polynomial $r_{j+1} p_{j+1}$ in our algorithm: see eq. (10). Paige's eq. (P4)

$$
r_{j+1}=\sqrt{\left(w_{j+1}, w_{j+1}\right)}
$$

is the same as eq. (25), computed in Lemma 2. En suite, eq. (P5),

$$
v_{j+1}=w_{j+1} / r_{j+1}, \quad(P 5)
$$

is nothing else than the normalization of the polynomial $p_{n+1}$. Finally, we have employed in the above Paige's eq. (P7), a crucial equality between two different coefficients in the recursions.

The attentive reader has surely noticed that our algorithm follows step by step the sequence of operations just proposed. Paige calls this the $A(1,7)$ version of Lanczos' algorithm, and shows its stability. Perhaps the most crucial remark in his error analysis is the fact that, regardless of cancellations which may occur in eq. ( $P 9)$, orthogonality between $v_{j}$ and $v_{j+1}$ is well preserved:

$$
r_{j+1}\left(v_{j+1}, v_{j}\right)=-r_{j}\left(v_{j-1}, v_{j}\right)+O(\varepsilon), \quad(P 10)
$$

so that $r_{j+1}\left|\left(v_{j+1}, v_{j}\right)\right| \leq 2(j+4) \varepsilon, \varepsilon$ being the arithmetical error [24].

In particular, Paige's analysis considers the case when the scalar products occurring in the previous equations are computed as ordinary scalar products of finite-dimensional vectors. This is also the case of our algorithm, as can be seen exposing its full equivalence, not only structural, but also computational, to Paige's $A(1,7)$.

To do this, let us define the space $\mathcal{L}=l_{2} \oplus l_{2} \oplus \cdots \oplus l_{2}$, where the number of terms in the direct sum is $M$ (the same as the number of I.F.S. maps). Vectors in this space will be denoted like $w=\left(w_{1}, w_{2}, \ldots, w_{M}\right), w_{i} \in l_{2}$. Define a scalar product $\langle\cdot, \cdot\rangle$ in $\mathcal{L}$ as:

$$
\left\langle w, w^{\prime}\right\rangle=\sum_{i=1}^{M} \pi_{i}\left(w_{i}, w_{i}^{\prime}\right)_{2},
$$

where $(\cdot, \cdot)_{2}$ denotes the usual scalar product in $l_{2}$, and $\pi_{i}$ are the I.F.S. maps weights. Finally, let $\Gamma_{i}^{n}$ and $\tilde{\Gamma}_{i}^{n}$ be the $l_{2}$ vectors whose components are $\Gamma_{i, l}^{n}$ and $\tilde{\Gamma}_{i, l}^{n}$ for $l=0, \ldots, n$ and null otherwhise. The letter $\Gamma^{n}$ without lower indices will be used to denote the $\mathcal{L}$ vector $\Gamma^{n}=$ $\left(\Gamma_{1}^{n}, \Gamma_{2}^{n}, \ldots, \Gamma_{M}^{n}\right)$, and likewise $\tilde{\Gamma}^{n}$.

First of all, notice that the normalization relation

$$
1=\sum_{i=1}^{M} \pi_{i}\left(\Gamma_{i}^{n}, \Gamma_{i}^{n}\right)_{2},
$$

following from the balance relation applied to $1=\int p_{n}^{2}(x) d \mu(x)$, can be written as

$$
1=\left\langle\Gamma^{n}, \Gamma^{n}\right\rangle \text {. }
$$

Next, notice that the computation of $A_{n}$ in eq. $(31,32)$ becomes, in matrix form,

$$
A_{n}=\sum_{i=1}^{M} \pi_{i}\left(\Gamma_{i}^{n},\left[\delta_{i} J+\beta_{i}\right] \Gamma_{i}^{n}\right)_{2}=\left\langle\Gamma^{n}, \mathcal{J} \Gamma^{n}\right\rangle,
$$


where $\mathcal{J}$ is the operator which acts as $\delta_{i} J+\beta_{i}$ in the $i$-th subspace. Eq. (37) is identical to eq. (P1). In fact, $\Gamma^{n}$ becomes here the Lanczos' vector $v_{n}$, and obviously $A_{n}$ are the diagonal matrix elements of the Lanczos' tridiagonalization of $\mathcal{J}$.

The next step in $A(1,7)$ is eq. $(P 9)$; for us, this is the updating relation of the coefficients $\Gamma$, which (Lemma 1) can be written as

$$
\tilde{\Gamma}^{n+1}=\mathcal{J} \Gamma^{n}-A_{n} \Gamma^{n}-r_{n} \Gamma^{n-1} .
$$

Further on, note that we have obtained $r_{n+1}^{2}$ via eqs. $(25,26)$ in Lemma 2 :

$$
r_{n+1}^{2}=\sum_{i=1}^{M} \pi_{i}\left(\tilde{\Gamma}_{i}^{n+1},\left[\delta_{i} J+\beta_{i}\right] \Gamma_{i}^{n}\right)_{2}:\left\langle\tilde{\Gamma}^{n+1}, \mathcal{J} \Gamma^{n}\right\rangle=\left\langle\tilde{\Gamma}^{n+1}, \tilde{\Gamma}^{n+1}\right\rangle .
$$

This is precisely Paige's eq. (P4)

Finally, eq. (P5) is the simple rescaling which leads from $\tilde{\Gamma}^{n+1}$ to $\Gamma^{n+1}$, and eq. (P7) is implicit, as above, in our formalism. We can therefore conclude that our algorithm is an exact version of Paige's $A(1,7)$, which is proven to be numerically stable.

\section{Experimental Stability Analysis of Different Algorithms}

In this section, we shall verify the predictions of sections IV and VI by applying directly the different solution algorithms to the determination of the Jacobi matrix in suitable test cases. We consider three different algorithms, based on:

O: The use of ordinary moments with a Cholewsky decomposition of the associated Gram matrix.

C: The use of Chebyshev moments (that is, sampling with the measure $d \sigma=\frac{1}{\pi \sqrt{1-x^{2}}} d x$ ) with a Cholewsky decomposition of the associated Gram matrix.

S: The direct Stieltjes algorithm proposed in section V.

As first test case, we choose the uniform measure on $[-1,1]$ associated with Legendre polynomials, for which classical theory gives $A_{n}=0$, and $r_{n}=\frac{n}{\sqrt{(2 n+1)(2 n-1)}}$. This measure is also the balanced measure (dilated and translated) of the I.F.S. with $M=2, \delta_{1}=\delta_{2}=\frac{1}{2}$, $\beta_{1}=0, \beta_{2}=\frac{1}{2}$, and $\pi_{1}=\pi_{2}=\frac{1}{2}$. Therefore, one can test algorithm $\mathrm{S}$ by comparing theory and numerical results. We ran these calculations in double precision on an IBM RS 6000/320. In Fig. 7 we plot the absolute errors in the determination of $A_{n}$ and $r_{n}$, as a function of $n$. The algorithm is found to be stable. Classical polynomial sampling is also known to be stable in this case.

As a second bench-mark, we choose the now familiar I.F.S. measure induced by the maps $\delta_{1}=\delta_{2}=\frac{2}{5}, \beta_{1}=0, \beta_{2}=\frac{3}{5}$, and $\pi_{1}=\pi_{2}=\frac{1}{2}$. Because of symmetry, we know that $A_{n}=\frac{1}{2}$, for all $n$, while the almost-periodic sequence $r_{n}$ is responsible for the singular character of the measure. In Fig. 8 we draw the errors in the determination of $A_{n}$ in double precision, and 
the absolute differences between double and quadruple precision results for $r_{n}$ obtained by algorithm S. The error propagation observed is consistent with a polynomial law with exponent close to four.

We can also employ algorithms $\mathrm{O}$ and $\mathrm{C}$, and compare the computed $r_{n}$ with the stable results obtained by algorithm S. The relative differences are plotted in Fig. 9: one can see that both techniques are ill-conditioned, and that the relative advantage offered by Chebyshev moments is limited to a smaller slope in the exponential growth of the error.

We can therefore conclude that the recursive algorithm presented in this paper effectively solves the problem of computing the Jacobi matrix associated with a balanced I.F.S. measure. Up to now, the sole technique which could cope with a reasonable polynomial order was a Padé procedure programmed in MAPLE by Vrscay [25]. Yet, it was limited by memory and time requirements to much smaller orders than those reachable by our numerical algorithm. Let us now turn to a first, immediate application of this technique.

\section{Integrating Fractal Measures}

The direct method for the determination of the Jacobi matrix proposed above can be used to solve the problem of integrating smooth functions with respect to an I.F.S. balanced measure: $I:=\int f(x) d \mu$. Up to now, the method of election to compute such integrals has been a form of ergodic theorem: If we take a realization of the random process $x_{k} \rightarrow \phi_{\sigma}\left(x_{k}\right):=x_{k+1}, \sigma$ being a random variable taking the integer values $i=1, \ldots, M$ with probabilities $\pi_{i}$, then the measure $\eta(x)=\frac{1}{N} \sum_{k=1}^{N} \delta\left(x-x_{k}\right)$ converges to $\mu$ in the weak* topology. That is to say, the sum $I_{N}:=\frac{1}{N} \sum_{k=1}^{N} f\left(x_{k}\right)$ converges to the integral $I$ for any continuous function $f$. The method allows large $N$ computations but its convergence is obviously slow.

A different method requires the diagonalization of the Jacobi matrix, and the use of Gauss formula (13). This method has not been profitable so far, because of the difficulty in computing high-order Jacobi matrices. A partial remedy was presented in the case of homogeneous I.F.S., for which an error formula, and a Richardson extrapolation procedure exist [26]. Our direct procedure permits to obtain the required integrals with the precision wanted.

To show this, let us consider the case of the homogeneous I.F.S. measure introduced by Bessis and Demko [21] to approximate the vibrational spectrum of a cubic, face-centered harmonic solid, and to the computation of the integral [27]

$$
I=\frac{1}{2} \int_{0}^{1} \sqrt{x} d \mu(x)
$$

where $d \mu(x)$ is an I.F.S. approximation to $G(x) d x$, the fraction of normal modes in $[x, x+d x]$. In [10] we showed that any measure on a compact set can be arbitrarily well approximated by I.F.S. measures; Bessis and Demko showed that this approximation can be very profitable in the particular case of eq. (40). We employ their four-map approximation [21] to compute the Jacobi matrix first, hence Gaussian points, and finally $I$. In Fig. 10 we plot $I_{n}$, the approximate integral with $n$ Gaussian points as a function of $n$. The rapid convergence of this technique is also seen computing the remainder $\Delta_{n}:=I_{n}-I$, as plotted in Fig. 11. This example shows that we are able to obtain extremely precise integrals at a polynomial computation price. 


\section{Conclusions}

We have presented a stable algorithm to compute Jacobi matrices associated to I.F.S. balanced measures. This algorithm consists of a recursive determination of Jacobi entries, in the framework of a Stieltjes approach. We have shown that this approach is demanded by the typical multi-fractal structure of these measures, which renders classical polynomial sampling ineffective. Thanks to the equivalence of our technique to the classical Lanczos procedure we have obtained a stability proof.

We can foresee various applications of our results, like e.g. to the integration of smooth functions, briefly discussed here, but also to the study of the asymptotic properties of the set of associated orthogonal polynomials, an important and challenging problem of analysis. In fact, a variety of results is known in the case of absolutely continuous measures (w.r.t. Lebesgue) [28], but little is known in the case of singular measures. Our results permit to attack this question in the class of I.F.S. measures.

Another question of particular relevance which can be studied in detail in this family belongs to the theory of Schrödinger operators: One might wonder what are the relations occurring between the character of the sequence of $A_{n}$ and $r_{n}$ coefficients (almost-periodicity of some kind to be precised) and the "fractality" of the related measure. These aspects will be touched in forecoming publications.

Finally, in a quantum mechanical context, the Jacobi matrices studied here can be employed as models of almost-periodic systems [29]: The dynamical properties of these systems can be studied in their essence, having extracted the essential information on the related spectral measures. The tools of analysis can then be applied to rephrase some physically significant problems, such as electron transport and delocalization of the quantum motion. In this latter problem, the techniques presented in this paper have lead us to significant results [30] which unveil the rôle of generalized dimensions in quantum mechanics.

\section{Acknowledgements}

Part of this work was completed at the Observatoire de Nice, thanks to a grant from Programma Galileo for Azioni integrate italo-francesi.

G.M. is also at Istituto Nazionale di Fisica della Materia, and at Istituto Nazionale di Fisica Nucleare, sez. di Milano.

\section{References}

[1] N.I. Akhiezer, The Classical Moment Problem, Hafner, (NY. 1965).

[2] J.A. Shohat and J.D. Tamarkin, The Problem of Moments, American Mathematical Society, (Providence R.I., 1963).

[3] W. Gautschi, Computational Aspects of Orthogonal Polynomials, in Orthogonal Polynomials, P. Nevai Ed., Kluwer (Dordrecht NL 1990), 181-216.

[4] R.A. Sack and A.F. Donovan, An Algorithm for Gaussian Quadrature Given Generalized Moments, Dept. of Maths Publ., Univ. of Salford, England (1969). 
[5] G.H Golub and J.H. Welsch, Calculation of Gauss Quadrature Rules, Math. Comp. 23 (1969) 221-230.

[6] W. Gautschi, On the Construction of Gaussian Quadrature Rules from Modified Moments, Math. Comp. 24 (1970) 245-260.

[7] J. Bellissard, D. Bessis, and P. Moussa, Chaotic States of Almost Periodic Schrödinger Operators, Phys. Rev. Lett. 49 (1982) 702-704.

[8] G.A. Baker, D. Bessis, and P. Moussa, A family of Almost Periodic Schrödinger Operators, Physica A 124 (1984) 61-77.

[9] M.F. Barnsley, J.S. Geronimo, and A.N. Harrington, Infinite-Dimensional Jacobi Matrices Associated With Julia Sets, Proc. Am. Math. Soc. 88 \# 4, (1983) 625-630.

[10] C.R. Handy and G. Mantica, Inverse Problems in Fractal Construction: Moment Method Approach, Physica D 43, (1990) 17-36.

[11] G. Mantica and A. Sloan, Chaotic Optimization and the Construction of Fractals, Complex Systems 3, (1989) 37-62.

[12] E.R. Vrscay and C.J. Roehrig, Iterated Function Systems and the Inverse Problem of Fractal Construction Using Moments, in Computers and Mathematics, E. Kaltofen and S.M. Watt Eds., Springer (Berlin, 1989).

[13] D. Bessis and G. Mantica, Construction of Multifractal Measures in Dynamics from their Invariance Properties, Phys. Rev. Lett. 66, (1991), 2939-2942.

[14] J. Hutchinson, Fractals and Self-Similarity, Indiana J. Math. 30 (1981) 713-747.

[15] P. Diaconis, M. Shahshahani, Products of Random Matrices and Computer Image Generation, Contemporary Mathematics 50 (1986) 173-182.

[16] M.F. Barnsley and S.G. Demko, Iterated Function Systems and the Global Construction of Fractals, Proc. R. Soc. London A 399 (1985) 243-275.

[17] M.F. Barnsley, Fractals Everywhere, Academic Press, (New York 1988).

[18] A. Zygmund, Classical Trigonometric Series, Cambridge Univ. Press, (Cambridge, 1968).

[19] E.R. Vrscay and D. Weil, Missing Moments and Perturbative Methods for Polynomial Iterated Functions Systems, Physica D 50, (1991) 478-492.

[20] C.W. Clenshaw, A Note on the Summation of Chebyshev Series, Math. Tables Aids Comput. 9 (1955) 118-120.

[21] D. Bessis and S. Demko, Stable Recovery of Fractal Measures by Polynomial Sampling, Physica D 47 (1991) 427-438. 
[22] T. J. Stieltjes, Quelques Recherches sur la Théorie des Quadratures dites Méchaniques, Oeuvres I, 377-396.

[23] C. Lanczos, An Iteration Method for the Solution of the Eigenvalue Problem of Linear Differential and Integral Operators, J. Res. Nat. Bur. Stand. 45 (1950) 255-282.

[24] C.C. Paige, Computational Variants of the Lanczos Method for the Eigenproblem, J. Inst. Maths Applics 10 (1972) 373-381.

[25] E.R. Vrscay, I.F.S. Theory and Applications and the Inverse Problem, in Fractal Geometry and Analysis, J. Bélair and S. Dubuc Eds., Kluwer, (Dordrecht, NL 1992) 405-468.

[26] S. Demko, Euler Maclauren Type Expansions for some Fractal Measures, in Fractals in the Fundamental and Applied Sciences, H.O. Peitgen, J.M. Henriques and L.F. Penedo Eds., Elsevier-North Holland (Amsterdam 1991) 101-110.

[27] J.C. Wheeler and R.G. Gordon, Rigorous Bounds for the Thermodynamic Properties of Harmonic Solids, J. Chem. Phys. 51 \# 12 (1969) 5566.

[28] W. Van Assche, Asymptotics for Orthogonal Polynomials and Three-Term Recurrences, in Orthogonal Polynomials, P. Nevai Ed., Kluwer (Dordrecht NL 1990) 435-462.

[29] D. Bessis and G. Mantica, Orthogonal Polynomials Associated to Almost Periodic Schrödinger Operators, J. Comp. Appl. Math. 48 (1993) 17-32.

[30] I. Guarneri and G. Mantica, Multifractal Energy Spectra and their Dynamical Implications, Phys. Rev. Lett. 3379 (1994) 3379-3382. 


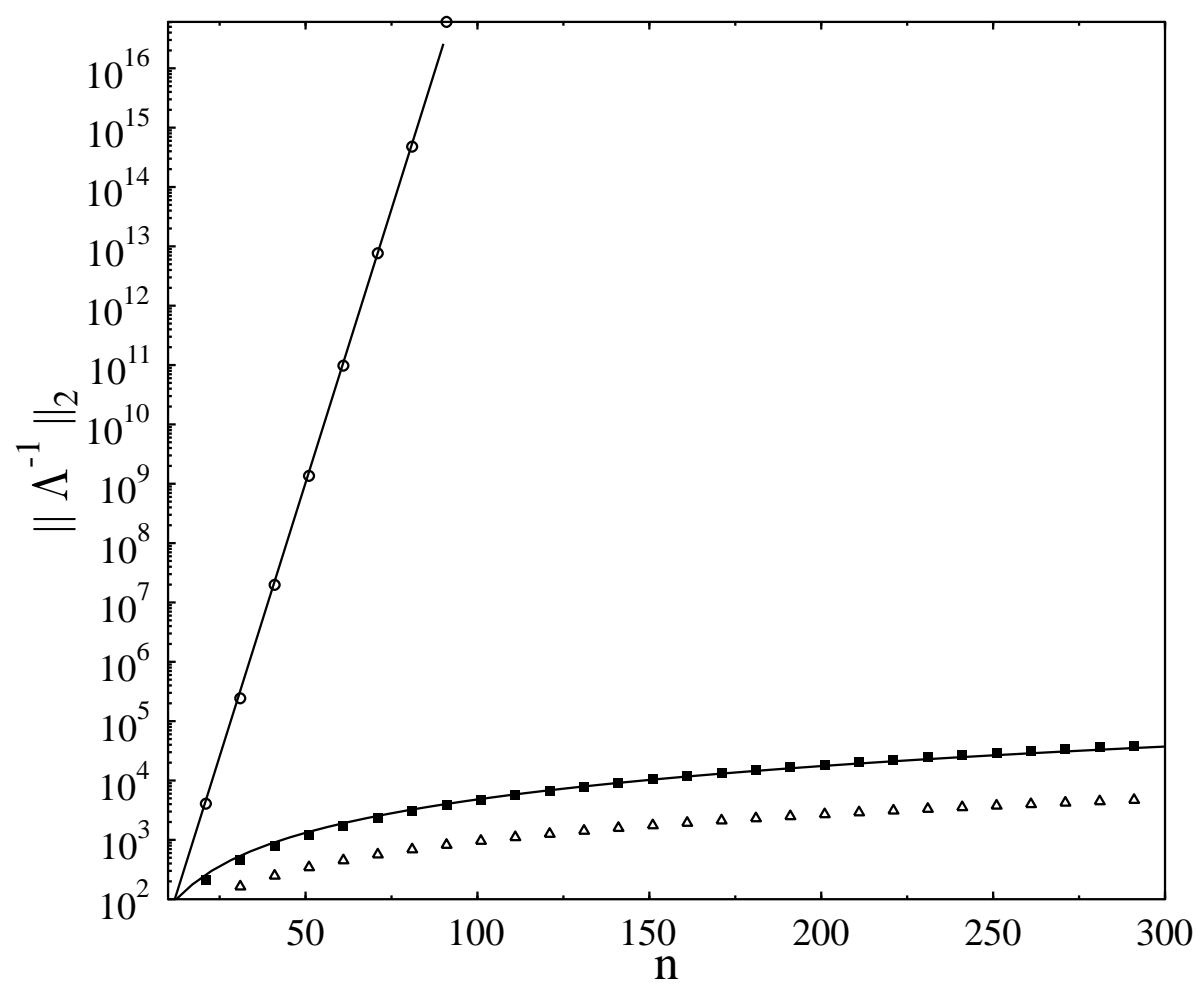

Figure 1: Euclidean norms $\left\|\Lambda^{-1}\right\|_{2}$ of the matrices defined in eq. (17), versus dimension, $n$. Three cases are reported: Chebyshev measure on $[-1,1]$, triangles; uniform measure on $[-1,1]$, filled squares, with interpolating power-law $n^{\eta}, \eta=1.87$; singular measure induced by the I.F.S. with maps $\left(\delta_{i}, \beta_{i}, \pi_{i}\right)=\left(\frac{2}{5}, 0, \frac{1}{2}\right),\left(\frac{2}{5}, \frac{3}{5}, \frac{1}{2}\right)$ rescaled to $[-1,1]$, circles. 


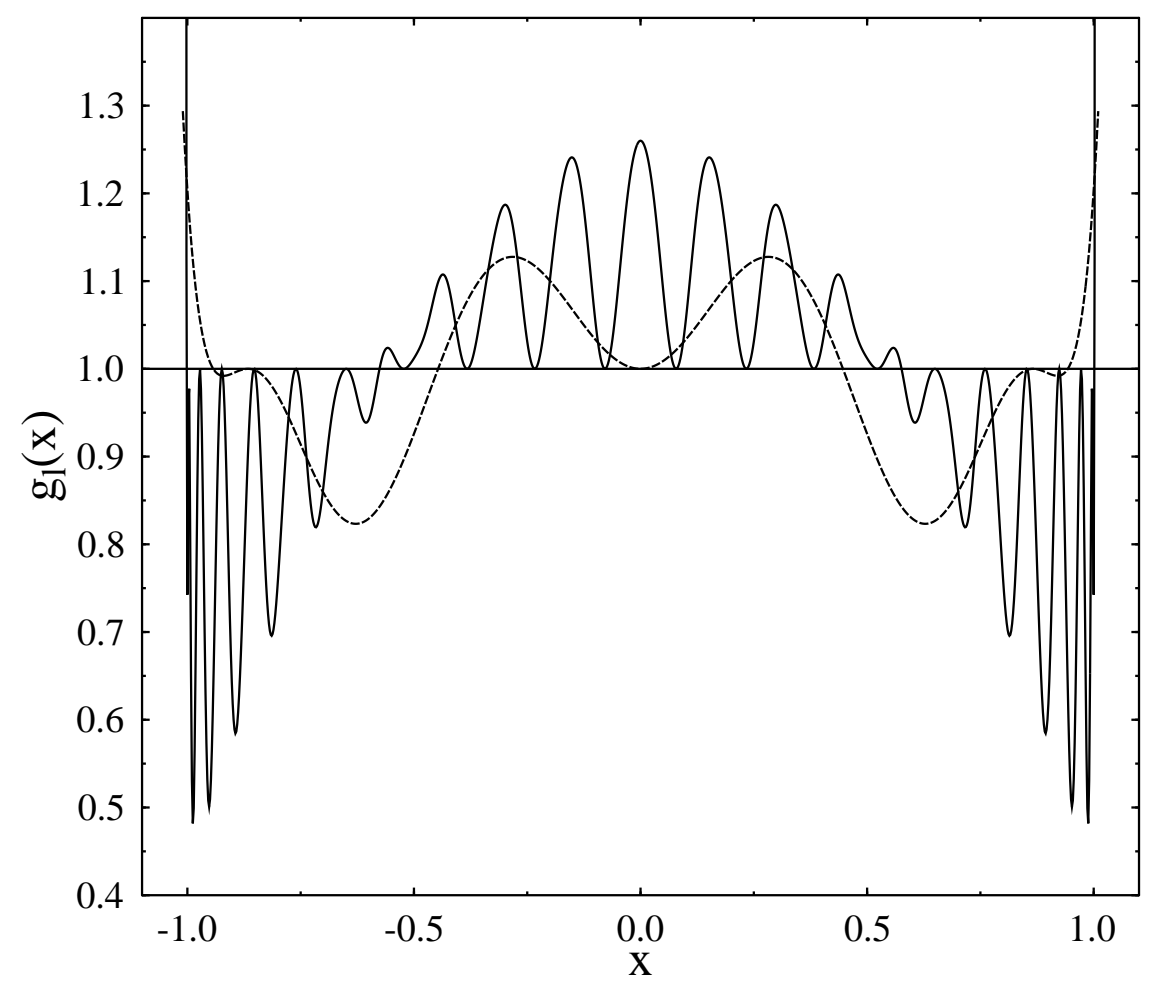

Figure 2: Interpolating polynomials $g_{l}(x)$ for the Chebyshev measure. The cases $l=3$ and $l=20$ are reported. The location of Gaussian points can be identified by tangencies to the horizontal line at unit height. 


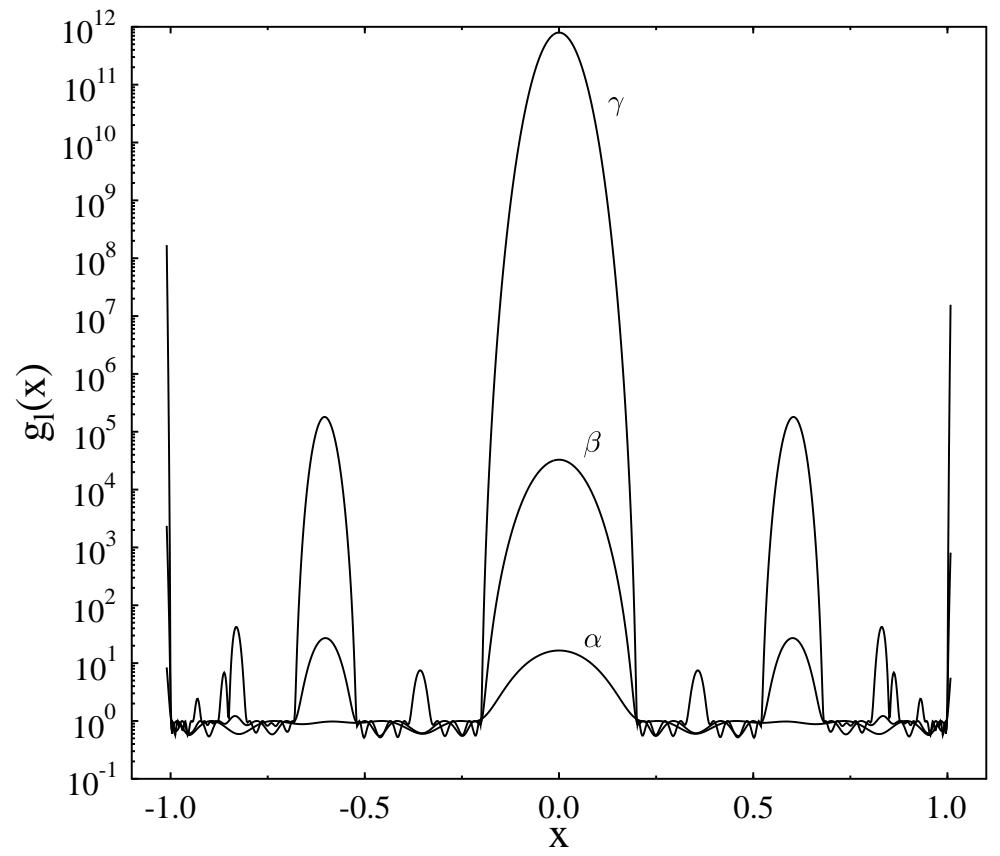

Figure 3: Interpolating polynomials $g_{l}(x)$ of the I.F.S. measure described in the text, for $l=10$ $(\alpha), l=20(\beta)$, and $l=40(\gamma)$. The vertical scale is here logarithmic, showing the rapid increase of polynomial values in the "gaps" of the support. 


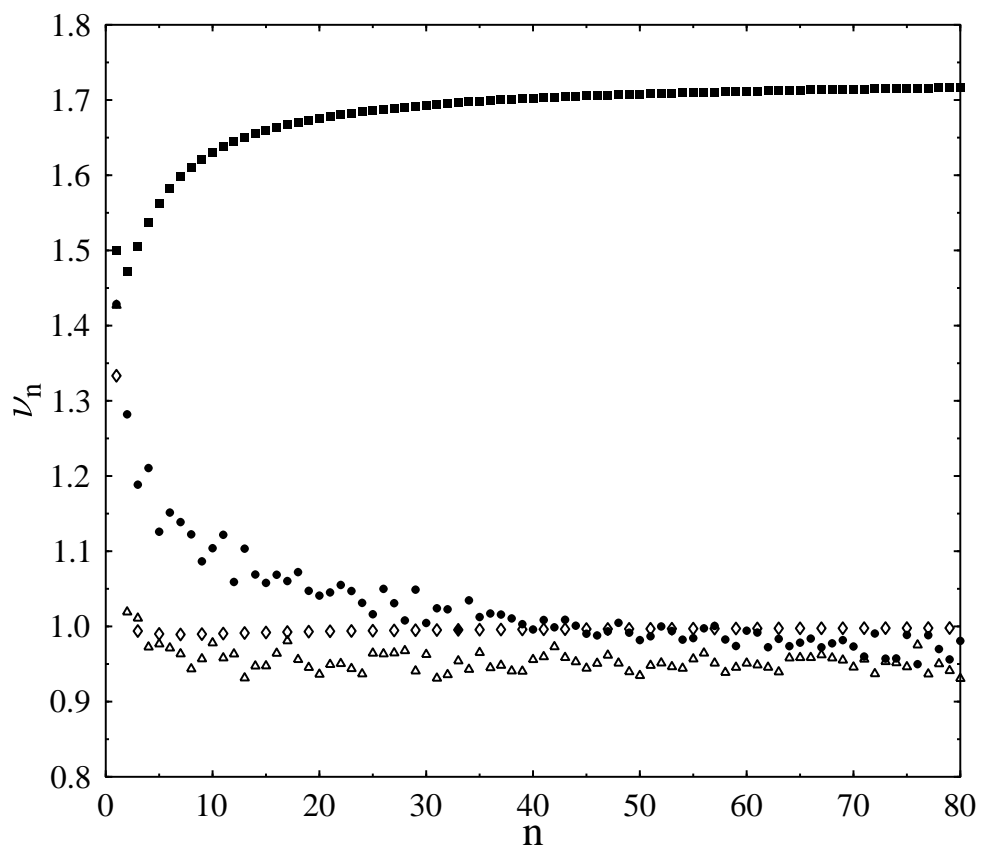

Figure 4: Sampling of classical measures: Frobenius norms $\nu_{n}$ of the $n$-dimensional matrix $\Xi^{-1}$, computed from the integral (19), versus $n$. The combinations sampler-sampled are: Chebyshev-Legendre (filled squares); I.F.S.-Legendre (filled circles); Legendre-Chebyshev (diamonds); I.F.S.-Chebyshev (triangles). See text for the other parameters. 


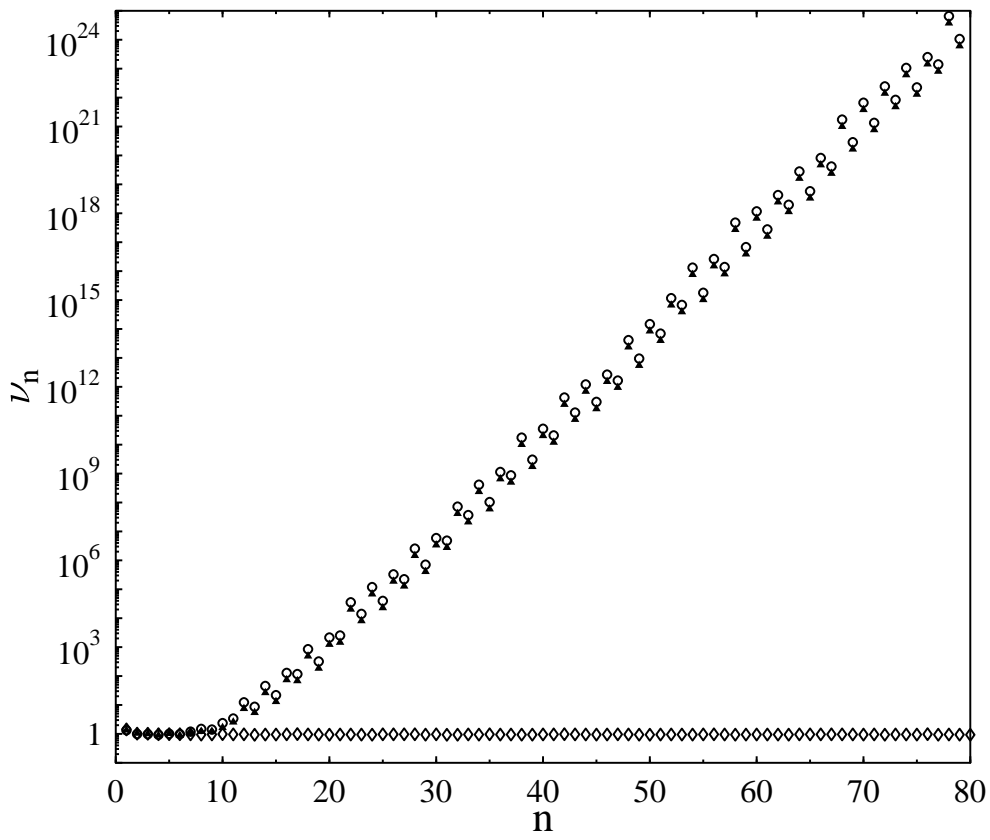

Figure 5: Sampling of a singular measure: as in Fig. 4. The combinations sampler-sampled are: Chebyshev-I.F.S. (filled triangles); Legendre-I.F.S. (circles); for comparison, we plot again the case I.F.S.-Chebyshev (diamonds) of Fig. 4. See text for the other parameters. 


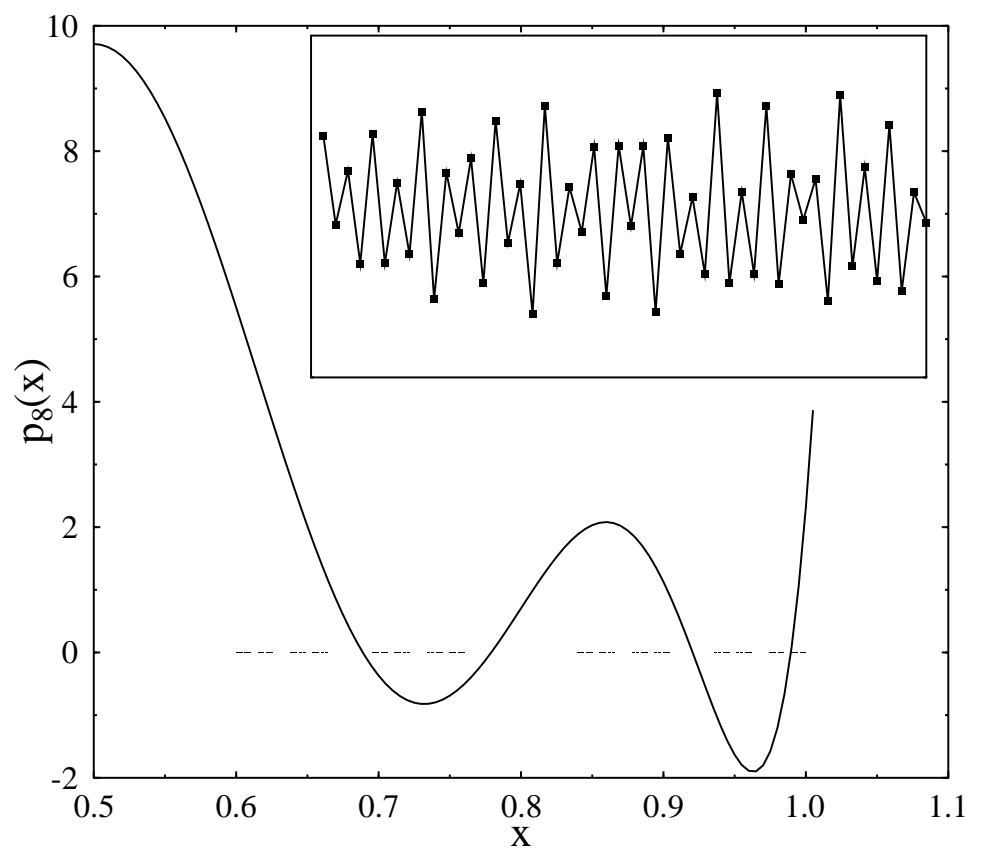

Figure 6: Orthogonal polynomial $p_{8}(x)$ of the I.F.S. measure with maps $\left(\delta_{i}, \beta_{i}, \pi_{i}\right)=\left(\frac{2}{5}, 0, \frac{1}{2}\right)$, $\left(\frac{2}{5}, \frac{3}{5}, \frac{1}{2}\right)$, with a finite-resolution representation of the support of the measure obtained by plotting a large number of points on the attractor. Because of the finite size of points, this latter appears as a sequence of dashes. In the frame, the sequence of $r_{n}$. The vertical scale ranges from zero to $\frac{1}{2}$. 


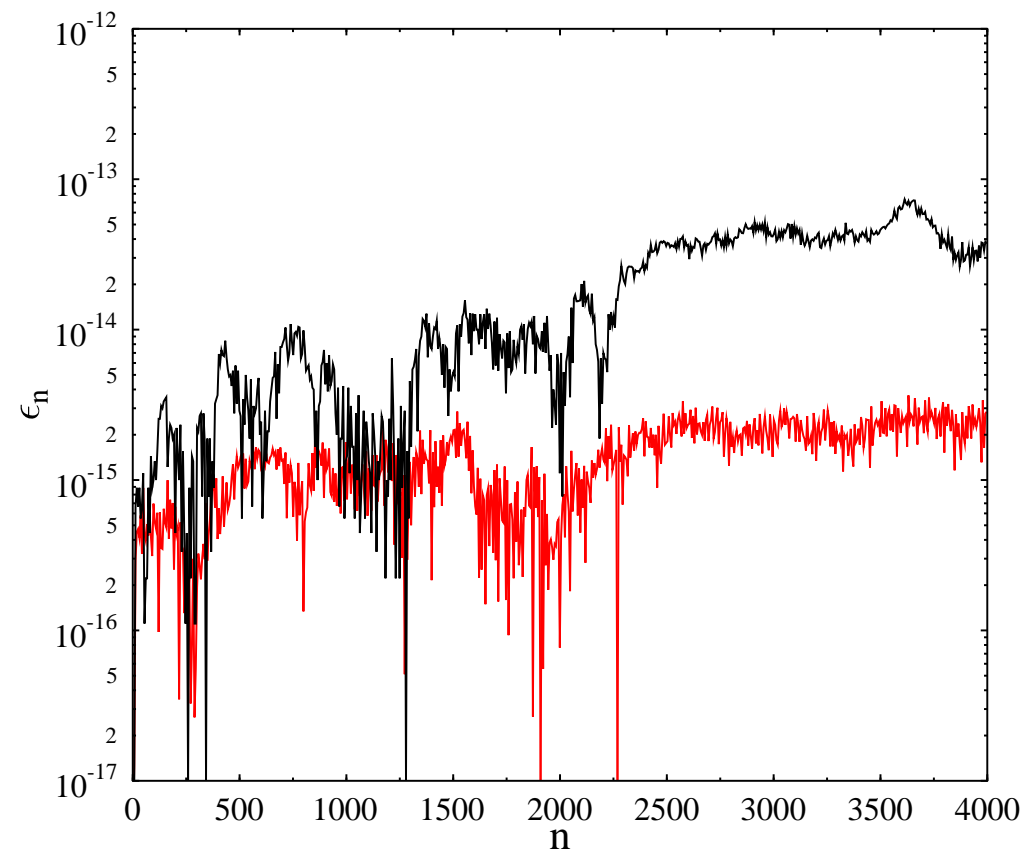

Figure 7: Absolute errors $\epsilon$ in the determination of $A_{n}$ (dashed curve) and $r_{n}$ (continuous), as a function of $n$, for the classical uniform measure. 


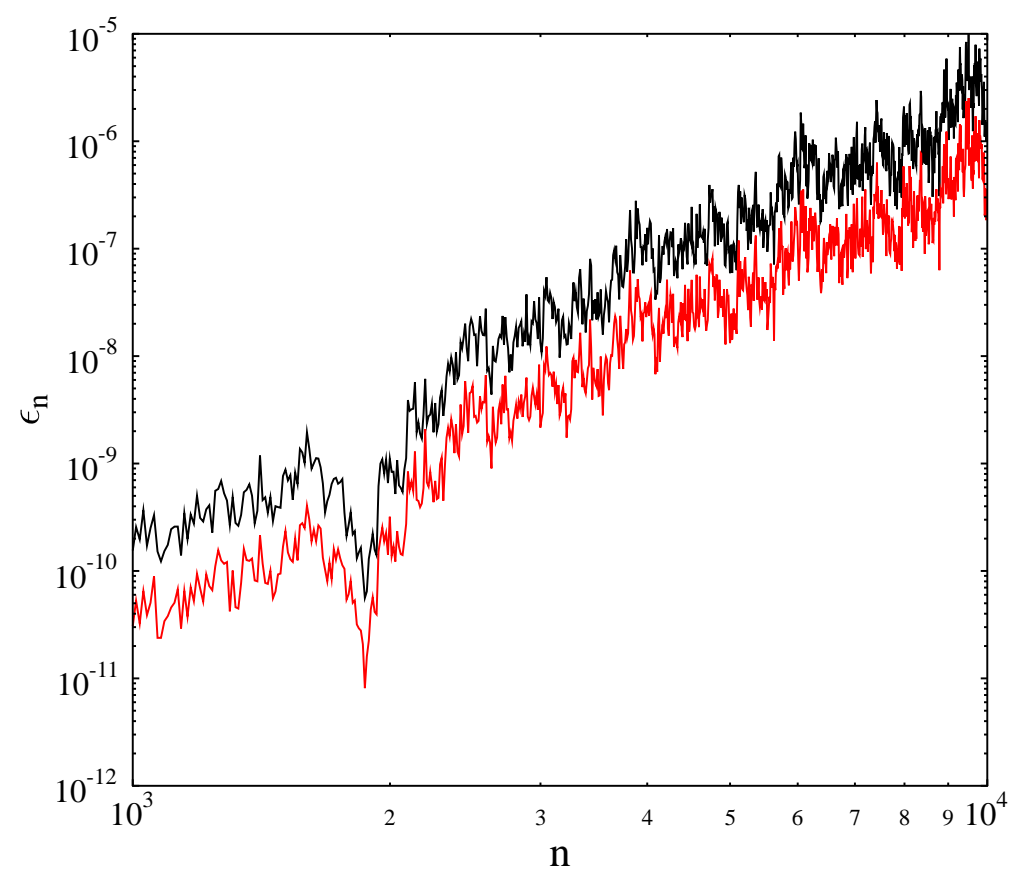

Figure 8: Absolute errors $\epsilon$ in the determination of $A_{n}$ (dark curve) obtained running algorithm $\mathrm{S}$ in double precision, and absolute differences in $r_{n}$ (light curve) between the results of $\mathrm{S}$ obtained in double and quadruple precision, versus $n$. See text for the other parameters. 


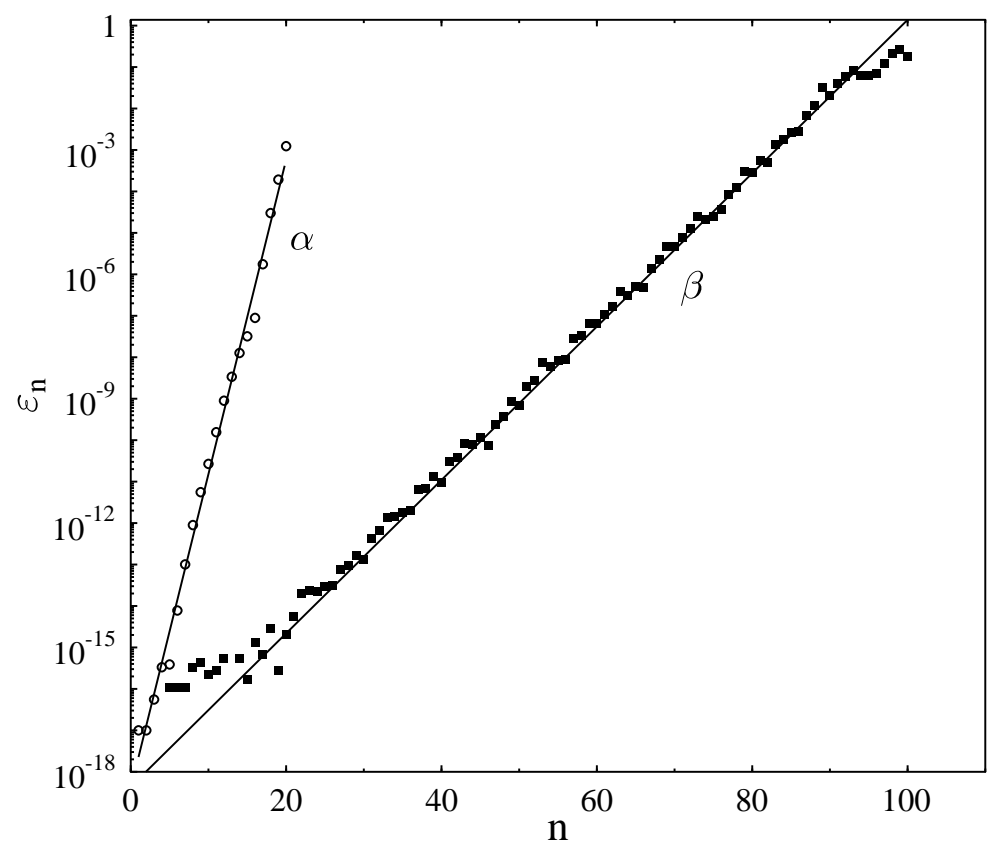

Figure 9: Absolute errors $\epsilon$ in the determination of $r_{n}$ effected by classical algorithms based on ordinary $(\alpha)$ and Chebyshev $(\beta)$ moments, for the I.F.S. measure described in the text. The slopes of the fitting lines are 1.75 and .426 , respectively. 


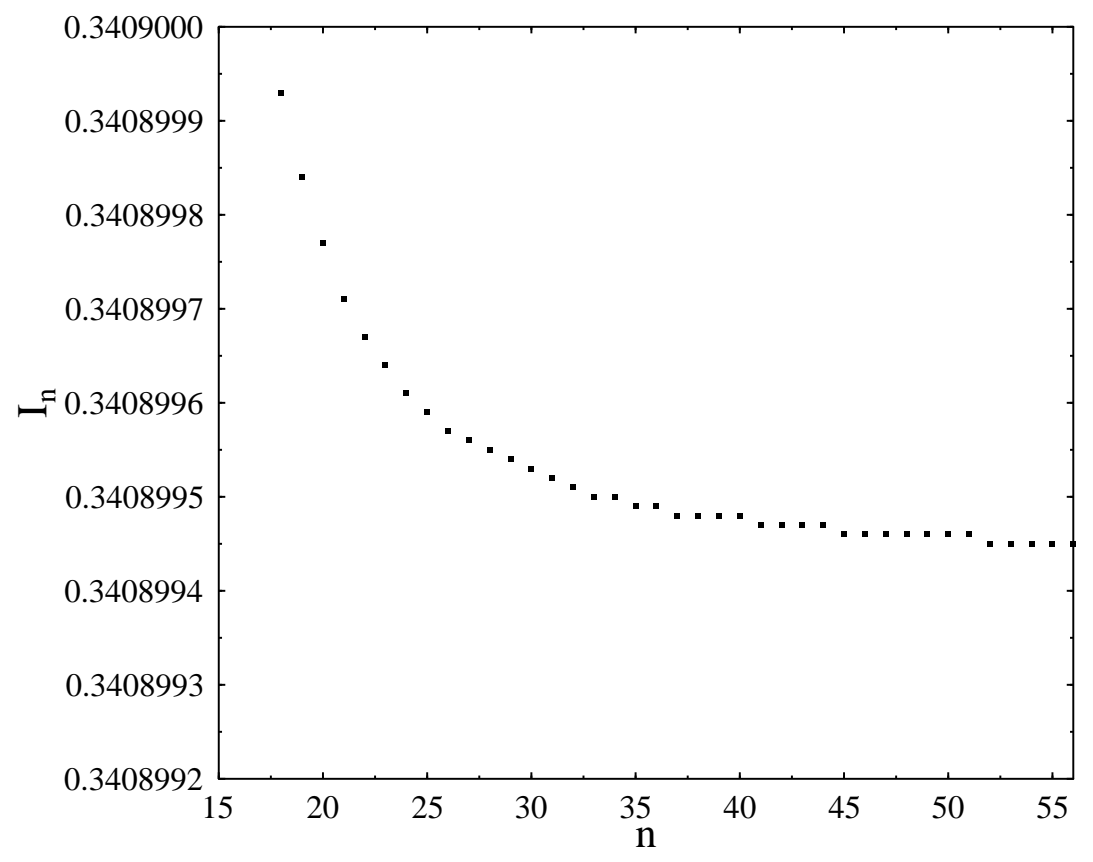

Figure 10: Results of the Gaussian integration of $I$ in eq. (40) versus the number of Gaussian points, $n$. 


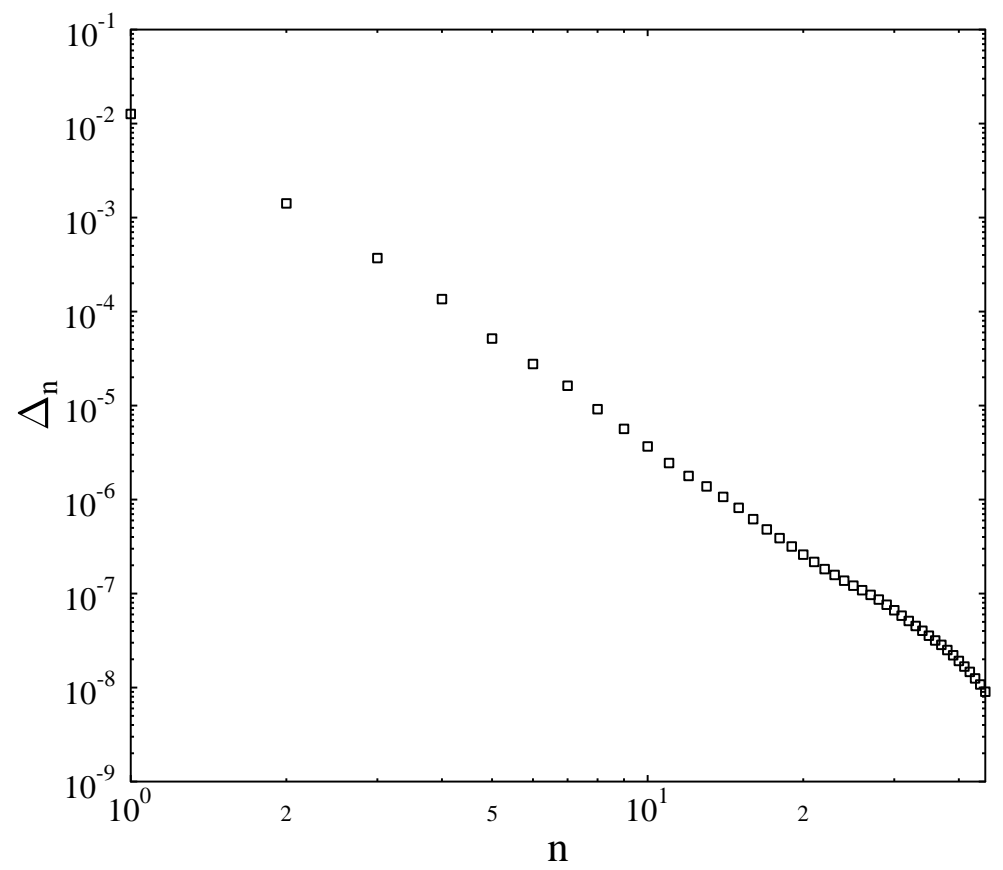

Figure 11: Difference between $I_{n}$ and the asymptotic result. See Fig. 10 and text. 